\title{
Encapsulation-Dependent Enhanced Emission of Near-Infrared Nanoparticles Using in vivo Three-Photon Fluorescence Imaging
}

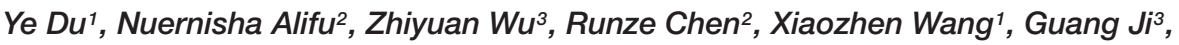

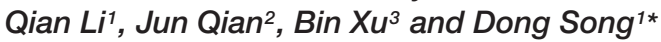

${ }^{1}$ Department of Breast Surgery, The First Hospital, Jilin University, Changchun, China, ${ }^{2}$ State Key Laboratory of Modern Optical Instrumentations, Centre for Optical and Electromagnetic Research, JORCEP (Sino-Swedish Joint Research Center of Photonics), Zhejiang University, Hangzhou, China, ${ }^{3}$ State Key Laboratory of Supramolecular Structure and Materials, Jilin University, Changchun, China

OPEN ACCESS

Edited by:

Jianxun Ding,

Changchun Institute of Applied Chemistry (CAS), China

Reviewed by:

Zhigang Xie,

Changchun Institute of Applied Chemistry (CAS), China

Xing Li,

Broad Institute, United States Jing Wen,

University of California, Los Angeles, United States

*Correspondence:

Dong Song

songdong690117@163.com

Specialty section: This article was submitted to Biomaterials,

a section of the journal Frontiers in Bioengineering and Biotechnology

Received: 08 July 2020

Accepted: 06 August 2020 Published: 10 September 2020

Citation:

Du Y, Alifu N, Wu Z, Chen R, Wang X, Ji G, Li Q, Qian J, Xu B and Song D (2020)

Encapsulation-Dependent Enhanced

Emission of Near-Infrared

Nanoparticles Using in vivo

Three-Photon Fluorescence Imaging.

Front. Bioeng. Biotechnol. 8:1029.

doi: 10.3389/fbioe.2020.01029
We discovered a unique fluorescent enhancement of dye encapsulated polymeric nanoparticles, which strongly depended on the polymeric matrix. Interestingly, the polymer nanoparticles containing a NIR emissive dye exhibited remarkable enhancement of emission encapsulated by the polymer amphiphilic polymer containing polystyrene (PS) moiety, whereas the nanoparticles showed weak fluorescence when using other polymer encapsulation. The highest fluorescent quantum yield of nanoparticles can reach $27 \%$ by using PS-PEG encapsulation, where the strong NIR fluorescence can be observed. These ultra-bright fluorescence nanoparticles also possess a strong three-photon fluorescence and show a good candidate for in vivo vascular three-photon fluorescence imaging of mouse brain and ear under $1550 \mathrm{~nm}$ fs laser excitation. A fine three-dimensional (3D) reconstruction with an imaging depth of 635 and $180 \mu \mathrm{m}$ was achieved, respectively. We further demonstrate that these nanoparticles can effectively target the sentinel lymph node (SLN) of mice.

Keywords: AIEgen, organic dye nanoparticles, NIR fluorescence, three-photon fluorescence, in vivo brain imaging

\section{INTRODUCTION}

Fluorescence imaging plays a very significant role in the fields of life science and biomedicine (Situ et al., 2016; Wang et al., 2017; Zhen et al., 2018)since it can offer high spatial resolution (Rust et al., 2006; Zhen et al., 2018) and sensitivity (Baddeley et al., 2011) to bio-samples. Deep tissue fluorescence imaging has attracted great research attention in clinical diagnosis and biomedical research, including functional brain imaging (Axelrod, 2001) sentinel lymph node mapping (Kobat et al., 2011; Qi et al., 2018; Zheng et al., 2018) and tumor targeting (Wu et al., 2017). The focusing ability and penetration depth of a light beam are limited by the optical absorption and scattering of biological tissue. Thus, the depth of fluorescence imaging is usually limited, which is a bottleneck in practical biomedical applications. Among these imaging techniques, near infrared (NIR) fluorescence imaging (Shao et al., 2015; Li et al., 2016; Song et al., 2016; Feng et al., 2017) and multi-photon excited fluorescence microscopy have become two deep-tissue fluorescence imaging approaches because of their potential abilities to overcome tissue absorption and scattering. Three-photon fluorescence microscopy, which has the advantages of a high signal-to-noise ratio, high penetration depth and spatial resolution, shines in the field of biological imaging. 
Over the past few decades, various fluorescent materials, such as semiconductor quantum dots, fluorescent carbon dots, metallic nanoclusters and organic fluorescent small molecules and nanoparticles, have been developed and extensively investigated for biological imaging applications (Liu et al., 2019). Among them, fluorescent polymer nanoparticles have shown great potential in bioimaging, diagnostics, drug delivery and therapy owing to their unique optical and electronic properties, easy functionalization and excellent biocompatibility (Qi et al., 2016; Ma et al., 2017). However, conventional organic dyes suffer from several problems, such as a small Stokes shift and aggregation-caused quenching (ACQ) effects (Qian et al., 2009; Massin et al., 2011; Wang et al., 2011; Lu et al., 2016; Fateminia et al., 2017). AIE molecules, which were discovered by Tang et al. (Liu et al., 2011; Shi et al., 2012), are organic compounds with excellent emission properties in the aggregated state or solid state that provide a novel approach to achieve high emission by manipulating the aggregated state. Recently, many kinds of AIE-based nanoparticles have been developed by using different methods to form fluorescent nanoparticles, such as AIEgens encapsulated into a polymer matrix and covalently binding AIEgens to polymers (Wang et al., 2013, 2016; Zhang et al., 2015; Yan et al., 2016). However, until now, reports of fluorescent nanoparticles, such as those with aggregation-dependent and fascinating luminescent properties, have been rare, probably due to the difficulty of molecular design and aggregation structure manipulation. High performance and expanded applications are therefore anticipated. Guidelines are available for specific molecular design to fine-tune the luminescent characteristics using a specific aggregation model for developing efficient fluorescent nanoparticles that can be utilized in biological imaging (Alifu et al., 2017; Qian and Tang, 2017). In this paper, we present a successful example of high-performance NIR fluorescent nanoparticles via manipulation of an encapsulation polymeric matrix based on the AIEgen 2-(4-bromophenyl)3-(4-(4-(diphenylamino)styryl)phenyl)fumaronitrile

(TB). Owing to the excellent optical characteristics, with both a high fluorescence quantum yield reaching $27 \%$ and strong threephoton fluorescence, these nanoparticles showed great potential applications for in vivo three-photon imaging and sentinel lymph node (SLN) mapping. Following such a strategy, additional nanoparticles with high-infrared fluorescence emission can be reasonably designed, which will provide more dye selection for fluorescent imaging.

\section{MATERIALS AND METHODS}

TB was synthesized according to our previous work (Alifu et al., 2017). PS-PEG and other chemical regents which not specially mentioned were purchased from Sigma Inc., The deionized water (DI water, $18.2 \mathrm{M} \Omega \mathrm{cm}$ resistivity) obtained from the system has been utilized in all the experiments Using a Shimadzu UV-3600 UV-vis spectrophotometer to measure UV-vis absorption spectra. Using a Malvern Zetasizer Nano ZS size analyzer to measure dynamic light scattering (DLS) and zeta potential at room temperature.
Fluorescence quantum yield was measured using a Hitachi F4500 spectrofluorophotometer. Rhodamine B (excitation wavelength: $365 \mathrm{~nm}$ ) was used as a standard to determine the fluorescence quantum yields of the nanoparticles.

\section{Preparation of the Nanoparticles (NPs)}

TB was dissolved in tetrahydrofuran (THF) to make a $1 \mathrm{mg} / \mathrm{mL}$ stock solution. Four polymers were dissolved in THF to form a $2 \mathrm{mg} / \mathrm{mL}$ polymer solution. First, $500 \mu \mathrm{L}$ of polymer solution (PS-PEG, PSMA, PIMA, F127) and 100, 66.7, 50, 40, and $33.3 \mu \mathrm{L}$ of the TB stock solution was placed into $2 \mathrm{~mL}$ Eppendorf tubes. Different amounts of THF were added to dilute to $1 \mathrm{~mL}$ uniformity with mixing and obtain mixtures with a weight ratio of TB to polymers of 1:10, 1:15, 1:20, 1:25 and 1:30. The mixture was rapidly added to $5 \mathrm{ml}$ deionized water, and the nanoparticles were formed after ultrasonic treatment. The THF was evaporated by continuous blowing nitrogen on a $78^{\circ} \mathrm{C}$ hot plate. A $220 \mathrm{~nm}$ filter head was used to obtain a transparent red $\mathrm{NP}$ aqueous solution.

\section{Cell Culture}

The cytotoxicity of TPABDFN-PSMA nanoparticles toward HeLa cells was evaluated by following the instructions of cell counting kit-8 (CCK-8). A total of 5000 cells/well in a $100 \mathrm{~mL}$ suspension were incubated in 96-well plates for $24 \mathrm{~h}$. Then, $100 \mathrm{~mL}$ of fresh culture medium containing PS-PEG@TB NPs at various concentrations (ranging from 0 to $120 \mu \mathrm{g} / \mathrm{mL}$ ) was added into each well. The cells were first incubated for $24 \mathrm{~h}$, then remove the medium, and the cells were washed with PBS for 3 times. Finally, $100 \mathrm{~mL}$ medium containing CCK-8 (10\%) was added to each well for $2 \mathrm{~h}$, and using a microplate reader (Thermo, United States) to measure its absorbance at $450 \mathrm{~nm}$.

\section{Measurement of the 3PL of PS-PEG@TB NPs}

The three-photon fluorescence spectrum of PS-PEG@TB NPs measured by the optical system is shown in Figure 3A. Adopting a $1550 \mathrm{~nm}$ femtosecond laser (FLCPA-01C, Calmar Laser, 400 fs, $1 \mathrm{MHz}$ ) as the light source. The laser beam was focused on a cuvette containing the PS-PEG@TB NP solution via a lens (focal length: $5 \mathrm{~cm}$ ). The $3 \mathrm{PL}$ was received vertically using an objective lens $(25 \times 1.05 \mathrm{NA})$ and then directed into the spectrometer (PG 2000, Ideaoptics Instruments) after filtering via a $980 \mathrm{~nm}$ short-pass filter.

\section{Optical System for 3PL Imaging}

The $1550 \mathrm{~nm}$ fs laser was coupled to an upright confocal microscope (Olympus, BX61W1-FV1000). After passing through a scan lens and a tube lens, the laser beam was focused onto the sample by a water-immersed microscope objective (XLPLN25XWMP2, Olympus, 25 1.05 NA). The imaging sample could be a glass capillary tube filled with an aqueous dispersion of PS-PEG@TB NPs or the brain or ear of a live mouse.3PL signals were epi-collected with the same objective and then passed through a customized $1035 \mathrm{~nm}$ short-pass dichroic mirror and a $590 \mathrm{~nm}$ long-pass filter (removing the excitation light and 
ambient noise). Then, the remaining fluorescence signals were collected using an external photomultiplier tube (HPM-100-50 Becker \& Hickl GmbH) via non-descanned detection (NDD). Pictures were collected every $5 \mu \mathrm{m}$ along the Z-axis, and $3 \mathrm{D}$ imaging was reconstructed by Z-scan stacks.

\section{In vivo Imaging of the Blood Vessels of the Mouse Brain}

Microsurgery of the cranial window on the mouse brain was performed. Briefly, the mice were anaesthetized, and a small piece of skull was excised using a dental drill. Microsurgery was performed under sterile conditions to avoid infection and damage. The mice were then intravenously injected with $200 \mathrm{~mL}$ of a PBS $(1 \times)$ solution of PS-PEG@TB NPs $(1 \mathrm{mg} / \mathrm{mL})$ and placed under the aforementioned multi-photon scanning microscope after being anaesthetized. To ensure that the mice could live well during the whole brain vascular imaging process, the body temperature of the mice was maintained at $37 \pm 1^{\circ} \mathrm{C}$ during the experimental period. For the description of the immobilization of mouse heads and how the objective of the upright multi-photon scanning microscope was arranged to contact the mouse brain, we refer to Qian's previous work (Qian et al., 2012). The 3PL signals of PS-PEG@TB NPs (from the brain blood vessels) were received using the 3PL imaging system.

\section{In vivo Imaging of the Blood Vessels of the Mouse Ear}

Three hundred milliliters of PS-PEG@TB NPs in $1 \times$ PBS solution $(1 \mathrm{mg} / \mathrm{mL})$ was intravenously injected into the mice. The mice were anesthetized and placed on a Petri dish with one ear attached to the coverslip and placed under the aforementioned optical system for $3 \mathrm{PL}$ imaging.

\section{SLN Mapping of Mice}

To investigate the SLN mapping of PS-PEG@TB nanoparticles in mice, we intradermally injected $0.1 \mathrm{~mL}$ nanoparticles (in $5 \%$ glucose) into the left forepaw pad of nude mice who were then anaesthetized with pentobarbital at various times after the NP injection. The sedated animals were then imaged using the in vivo optical imaging system.

\section{RESULTS AND DISCUSSION}

\section{Preparation and Characterization of PS-PEG@TB NPs}

TB could be synthesized by our previously reported method whose structure was fully verified by $1 \mathrm{H}$ NMR spectroscopy and high-resolution mass spectroscopy (HRMS) (Supplementary Figures S1, S2). As shown in Figure 1A, the TB has two main absorption peaks, mainly due to the $\pi-\pi^{*}$ transition (Figure 1A). The emission peak of TB reaches the NIR region, which is conducive to biological imaging (Figure 1B; Qian et al., 2012). By taking advantage of the amphiphilic properties of the copolymer, a water-dispersed polymeric micelle can be formed via self-assembly in aqueous solution (Scheme 1).
Notably, the nanoparticles doped with TB showed weak emission in solution when encapsulated with the amphiphilic polymer F127. Moreover, the fluorescent quantum yield was approximately $1 \%$ when the concentration of dye was changed. This is quite different from most reported AIEgens, which exhibit strong emission after polymeric encapsulation (Ni et al., 2018). However, to our surprise, the nanoparticles showed strong emission when we used another amphiphilic copolymer, PSMA, containing a polystyrene moiety. The nanoparticles showed excellent optical properties, including a maximum absorption at $473 \mathrm{~nm}$, the emission peaking at $671 \mathrm{~nm}$, and more importantly, the fluorescent quantum yield can reach $20 \%$ by optimizing the ratio of dye to copolymer (Table 1 ). In addition, the luminescent feature of the nanoparticles encapsulated by PIMA was similar to those encapsulated by F127, which only changed the polystyrene moiety to polyisobutylene. As shown in Table 1, the fluorescent quantum yield of PIMA@TB nanoparticles showed a slight increase to $5 \%$, which is still much lower than that of the PSMA@TB nanoparticles. These observations show that $\mathrm{TB}$ presents a unique luminescent feature when encapsulated by various amphiphilic copolymers. It is worth noting that the main difference between these copolymers is the benzene unit on the side chain of the polystyrene moiety, which shows more hydrophobic properties and larger steric hindrance than flexible alkyl and alkoxy chains. When $T B$ was mixed in a soft polymer matrix, the movement of the polymer segments at room temperature and relatively large free volume between the polymer chains enable the intramolecular motions of $T B$ in the aggregate state. These intramolecular motions can consume excited-state energy and lack emission of $T B$. Because $\mathrm{TB}$ is a multi-aromatic organic compound, the benzene unit may help the dye ТВ disperse well in the polymeric matrix due to moderate interactions between the polymer side chain and TB. These intramolecular motions can be restricted when $T B$ was dispersed in a rigid polymer matrix, allowing the molecules to decay via radiative channels and show strong emission. The proposed mechanism of AIE behavior is the restriction of intramolecular motions (RIM), which has been demonstrated in many typical AIE system. Therefore, we suggest that TB shows an encapsulation-dependent enhanced emission feature, whose luminescent properties strongly depend on the side chain of the amphiphilic copolymer. In particular, the nanoparticles encapsulated by the polystyrene-containing copolymer boost efficient NIR emission, whereas the fluorescence of the nanoparticles was very weak with low efficiency. To demonstrate our hypothesis, we further fabricated nanoparticles encapsulated by the amphiphilic copolymer PS-PEG, which contains polystyrene and PEG segments. We optimized nanoparticles encapsulated with different polymers (Supplementary Figures S3-S6). Notably, the highest fluorescent quantum yield of $27 \%$ was reached when we optimized the doping concentration of the dye as well as the ratio of polymer to dye (Supplementary Figure S7). The blue-shifted emission of nanoparticles encapsulated by PSMA and PS-PEG may originate from the enhanced intermolecular interactions between the benzene ring of polymer and $T B$, resulting the more 

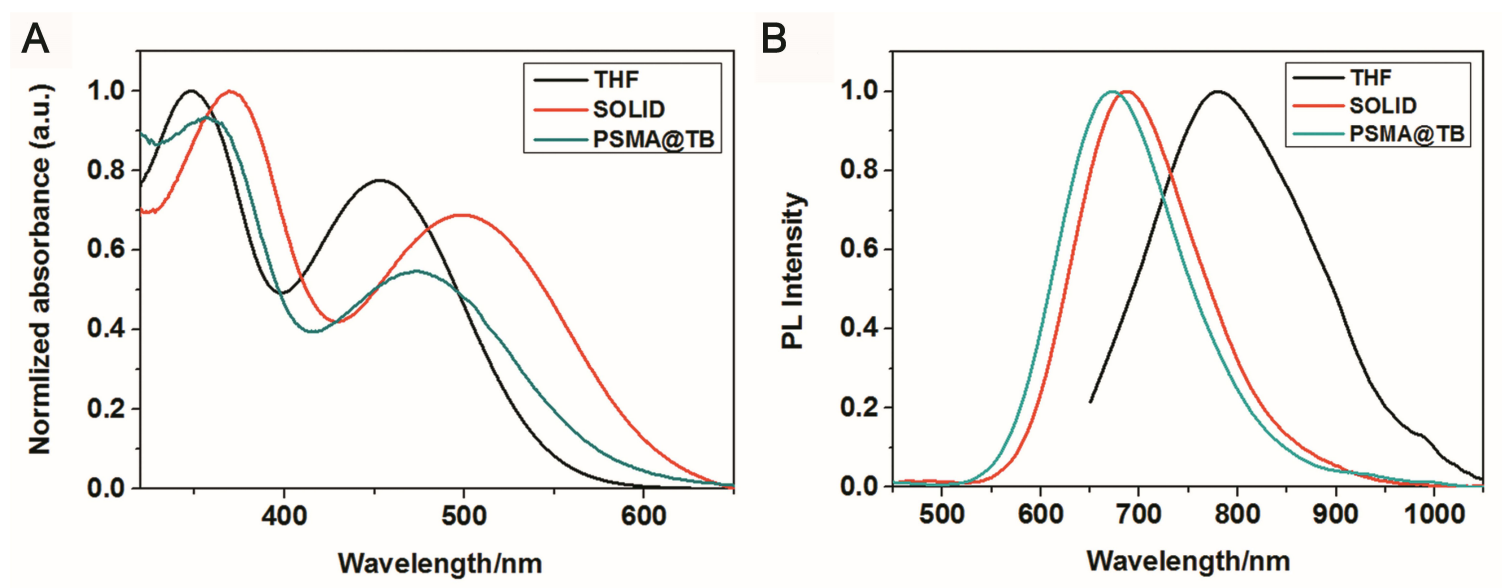

FIGURE 1 | (A) UV-vis absorption and (B) PL emission spectra (excitation wavelength: $365 \mathrm{~nm}$ ) of TB in THF (10-5 M) and in solid form and encapsulated with PSMA.
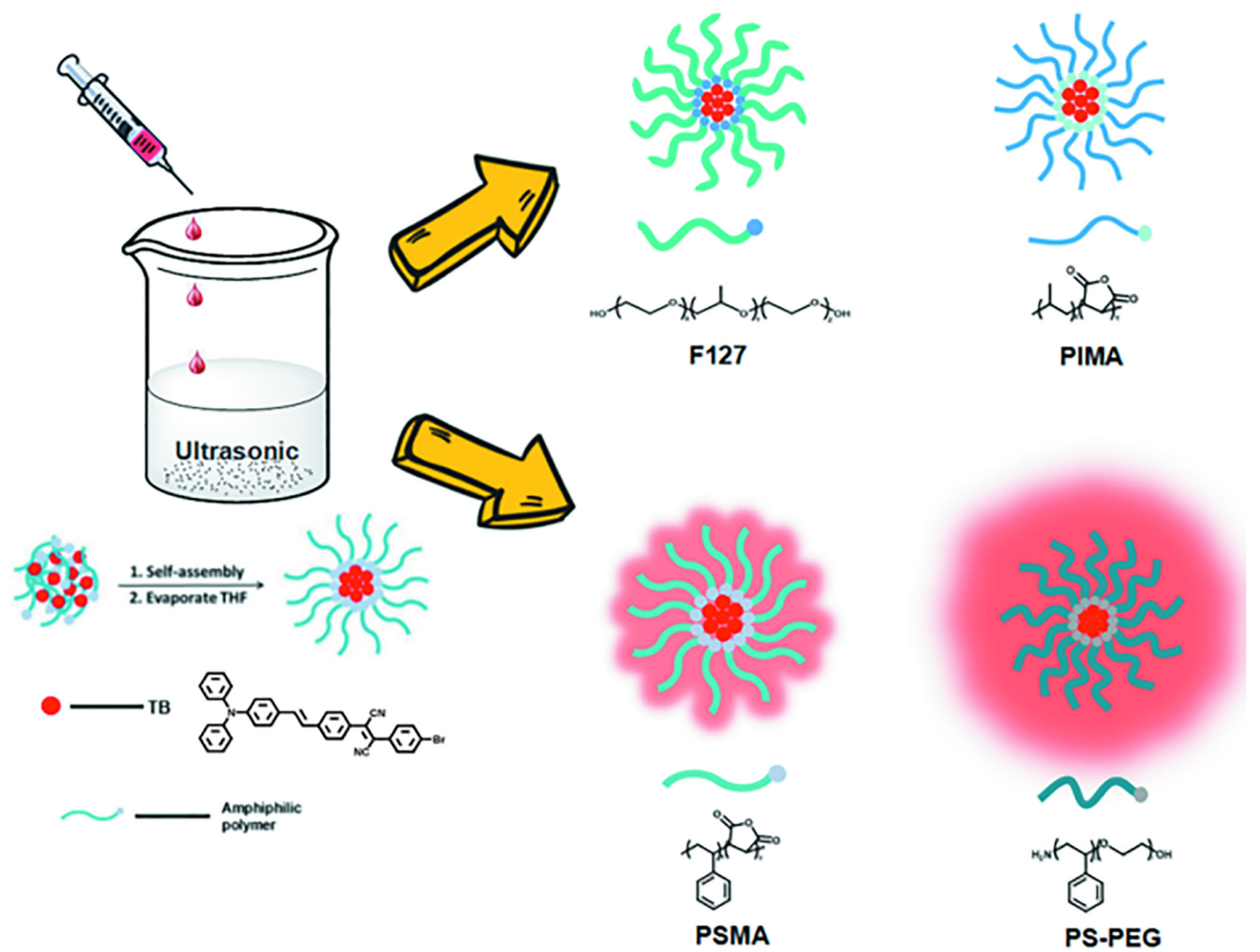

PIMA

SCHEME 1 | Schematic illustration of the preparation of TB loaded NPs.

twisted molecular conformation in the hydrophobic part. As shown in Figure 2, the nanoparticles present some excellent optical properties, such as a maximum absorption at $483 \mathrm{~nm}$ and emission peaking at $665 \mathrm{~nm}$. In addition, the $\tau$ of nanoparticles is 4.26 ns (Supplementary Figure S8) and the nanoparticles showed good dispensability with an average diameter of approximately $60 \mathrm{~nm}$ (Supplementary Figure S9) and an apparent zeta potential of -12.5 mV (Supplementary Figure S10), which are beneficial to biological imaging applications34 (Ni et al., 2018). 
TABLE 1 | Fluorescence quantum efficiency with different polymer proportions

\begin{tabular}{lcccc}
\hline $\begin{array}{l}\text { Different weight } \\
\text { ratio (TB: } \\
\text { copolymer) }\end{array}$ & $\boldsymbol{\Phi}$ F127@TB & $\boldsymbol{\Phi}$ PIMA@TB & $\boldsymbol{\Phi}$ PSMA@TB $\boldsymbol{\Phi}$ PS-PEG@TB \\
\hline $1: 10$ & 1.3 & 5.0 & 13.9 & 8.2 \\
$1: 15$ & 1.2 & 5.6 & 14.7 & 17.7 \\
$1: 20$ & 1.1 & 4.5 & 18.1 & 20 \\
$1: 25$ & 1.3 & 4.4 & 20.0 & 27.1 \\
$1: 30$ & 1.3 & 4.2 & 19.6 & 21.5 \\
\end{tabular}

\section{Cell Viability Analysis}

Supplementary Figure S11 shows the relative viabilities of HeLa cells treated with PS-PEG@TB NPs after $24 \mathrm{~h}$. The cells remained over $90 \%$ viable even when the concentration of the PS-PEG@TB NPs reached $120 \mu \mathrm{g} / \mathrm{mL}$, indicating the low cytotoxicity of PS-PEG@TB NPs and further validating their application in bioimaging.

\section{In vivo 3PL Imaging of Mouse Ear Blood Vessels}

Owing to the excellent NIR luminescent properties of PS-PEG@TB, we explored their application in a home-built multi-photon system to record the non-linear optical response of the PS-PEG@TB NPs under the excitation of a $1550 \mathrm{~nm}$ fs laser. Bright 3PL was observed, accompanied by sharp third harmonic generation (THG), as shown in Figure 3A. The 3PL spectrum of PS-PEG@TB NPs was centered at $655 \mathrm{~nm}$, giving a deep red emission. The inset in Figure 3A shows 3PL imaging of an aqueous dispersion of PS-PEG@TB NPs in a glass capillary tube under $1550 \mathrm{~nm}$ fs laser excitation. Red fluorescence can be clearly observed. The power dependence relationship of the PS-PEG@TB NPs was studied under $1550 \mathrm{~nm}$ fs excitation. As shown in Figure 3B, the fluorescence intensity of PS-PEG@TB NPs had a very good linear relationship to the cubic of the excitation intensity, indicating that 3PL would be the main nonlinear optical process. First, we used a mouse ear blood vessel model to examine the capability of PS-PEG@TB NPs in in vivo 3PEFM imaging. Figures 4A-F shows the images of PS-PEG@TB NPs at various depths of the mouse ear skin under $1040 \mathrm{~nm}$-fs excitation. Aside from the small capillaries located throughout the dermis, major veins and arteries located deeper within the dermis could also be observed. The overall distribution of blood vessels can be observed by stacking the images as shown in Figure 4G. Figure $4 \mathrm{H}$ shows a 3D reconstructed image of the blood vasculature network within a region of the ear dermis. Otherwise, the fluorescent intensity of PS-PEG@TB nanoparticles under the $1550 \mathrm{~nm}$ laser irradiation show a little reduction over $90 \mathrm{~min}$, demonstrating that PS-PEG@TB have excellent photostability as

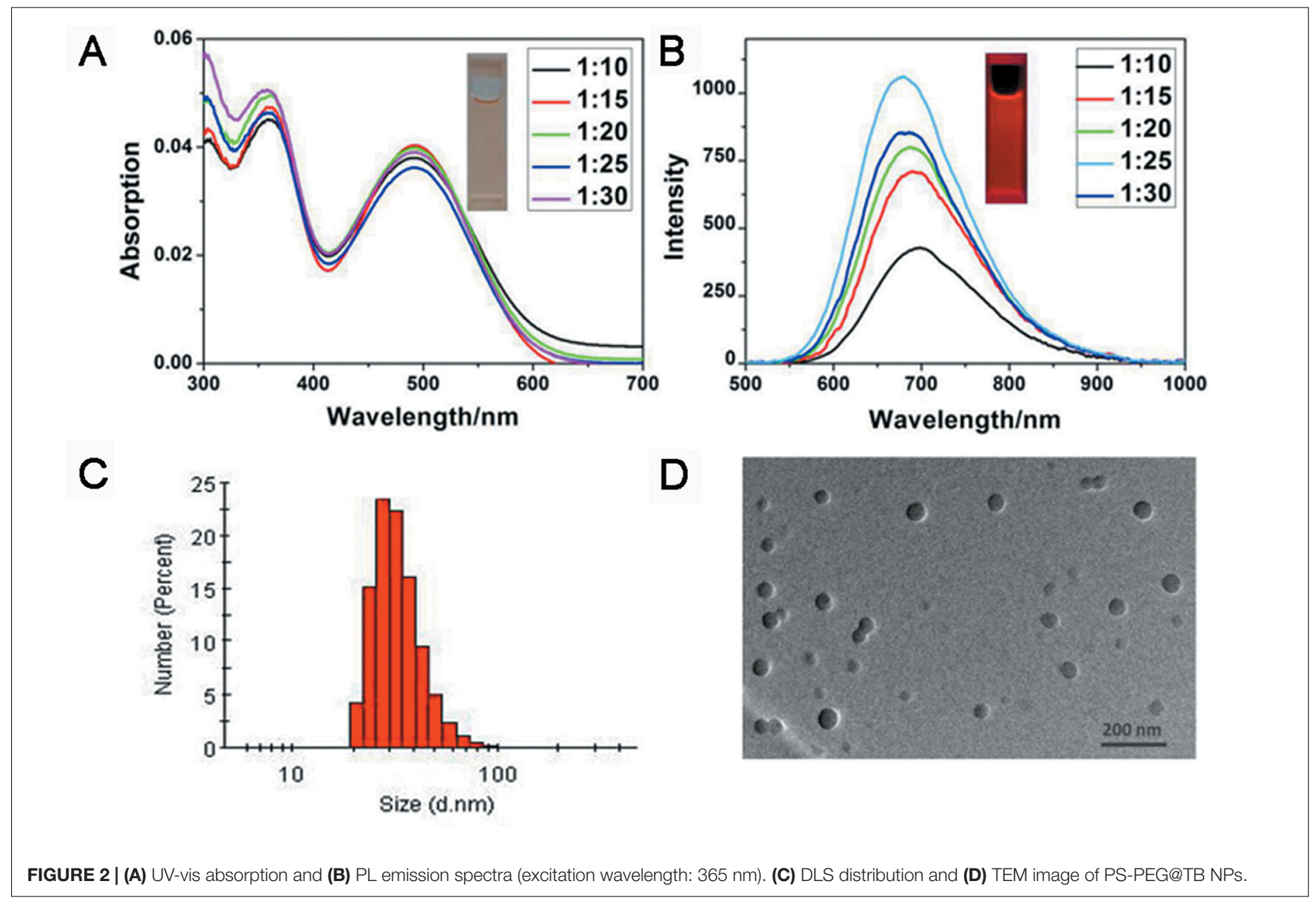



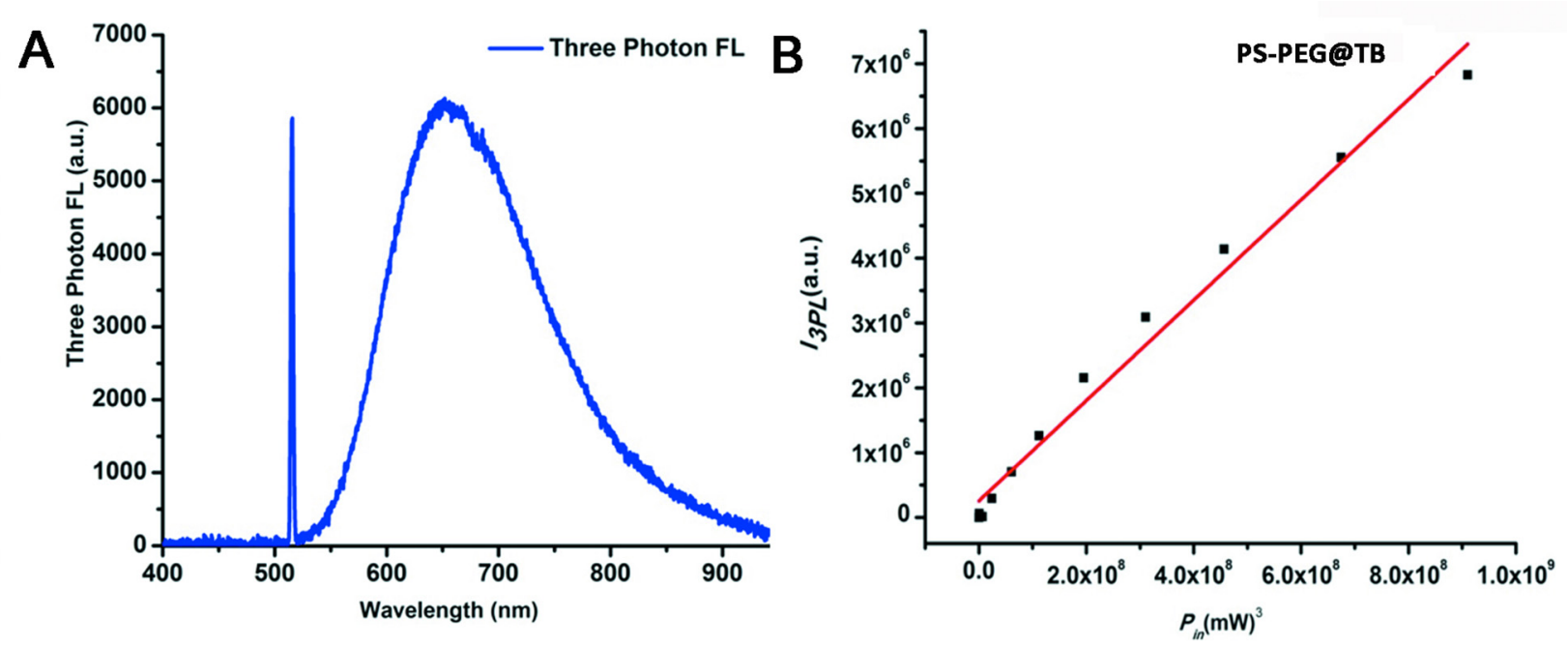

FIGURE 3 | (A) Three-photon fluorescence spectra of PS-PEG@TB NPs under 1550 nm fs excitation. (B) Cubic dependence of the three-photon-induced fluorescence of PS-PEG@TB NPs on the excitation intensity of the 1550 nm-fs laser.

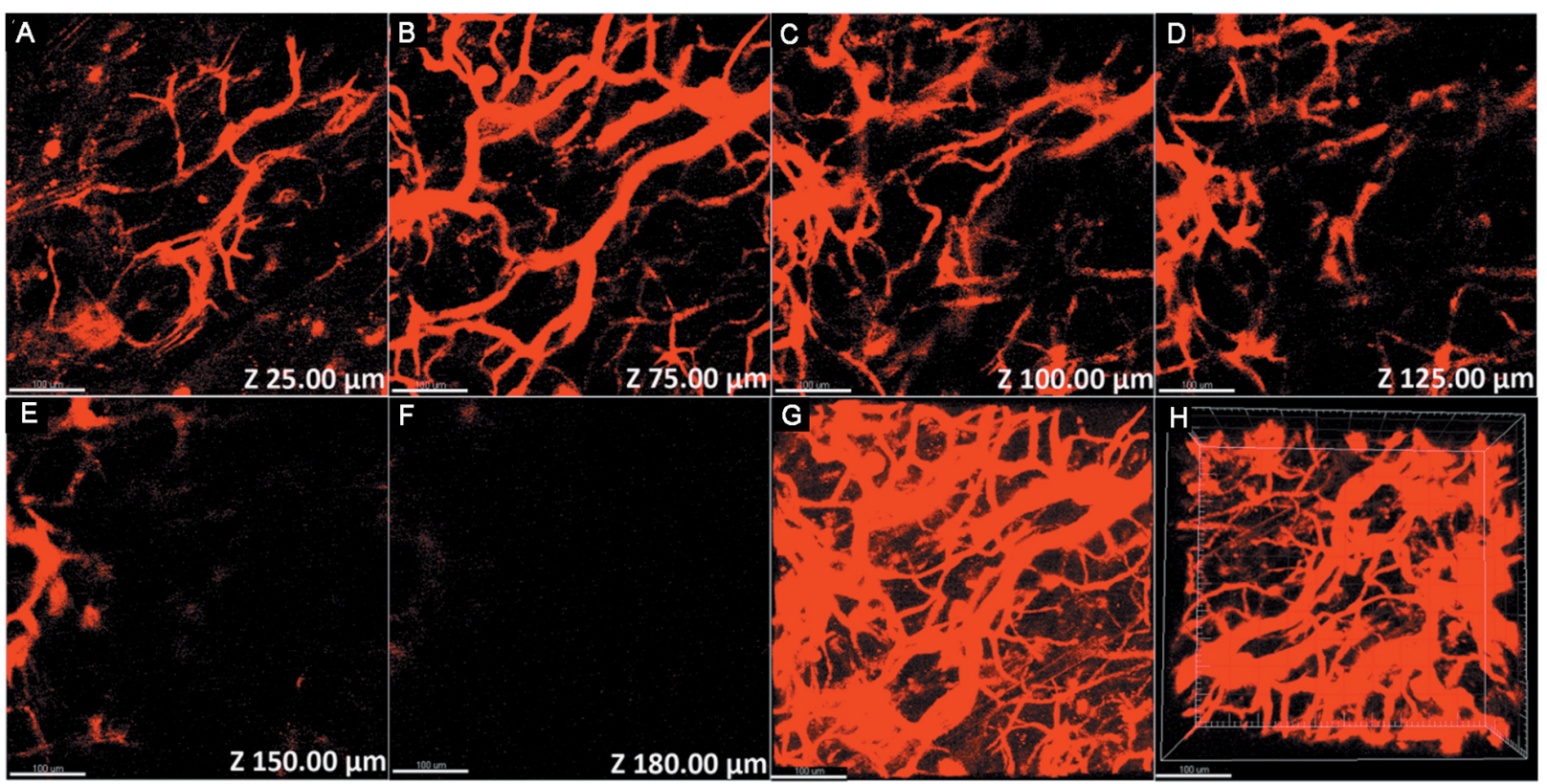

FIGURE 4 | 3PL imaging of ear blood vessels of a mouse treated with PS-PEG@TB NPs at various vertical depths: (A) $25 \mu \mathrm{m}$, (B) $75 \mu \mathrm{m}$, (C) $100 \mu \mathrm{m}$, (D) $125 \mu \mathrm{m}$, (E) $150 \mu \mathrm{m}$, and (F) $180 \mu \mathrm{m}$. (G) A stacked three-photon fluorescence image from a depth of 0 to $180 \mu \mathrm{m}$. (H) A 3D reconstructed image showing the distribution of the PS-PEG@TB NPs in the ear blood vessels of the mouse. Scale bar: $100 \mu \mathrm{m}$.

shown in Supplementary Figure S14. These results indicated that PS-PEG@TB NPs hold great promise as an alternative contrast agent for intravital blood vasculature imaging.

\section{In vivo 3PL Imaging of Mouse Brain Blood Vessels}

PS-PEG@TB NPs were further applied for the in vivo imaging of mouse brain blood vessels under the excitation of a $1550 \mathrm{~nm}$ fs laser. As shown in Supplementary Figure S13, PS-PEG@TB NPs emitted a bright 3PL signal in mouse brain blood vessels. As shown in Supplementary Figures S12A-G, the 3PL signal of PS-PEG@TB NPs clearly exhibit the structure of the mouse brain blood vessels at different depths, showing capillaries and even some of the fine structure. At imaging depths from 0 $\mu \mathrm{m}$ (Supplementary Figure S12A) to $385 \mu \mathrm{m}$ (Supplementary Figure S12D), there are some mainly coarse vessels. At imaging depths from $485 \mu \mathrm{m}$ (Supplementary Figure S12E) to $635 \mu \mathrm{m}$ 
A

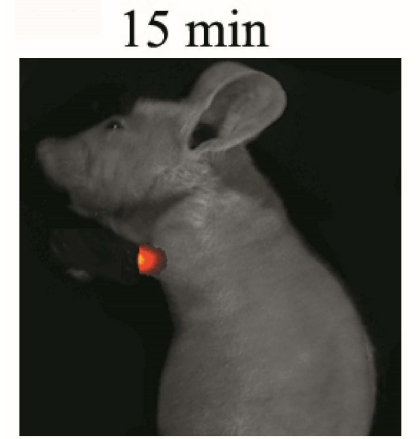

$60 \mathrm{~min}$

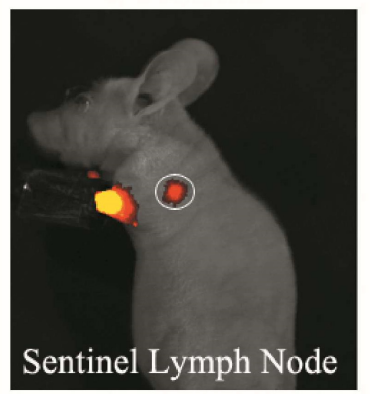

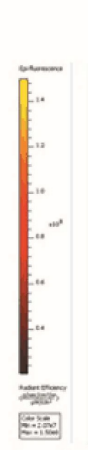

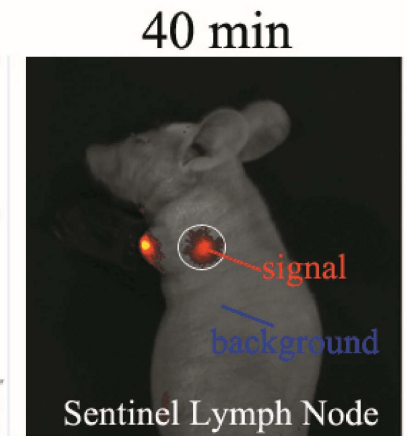

\section{$90 \mathrm{~min}$}
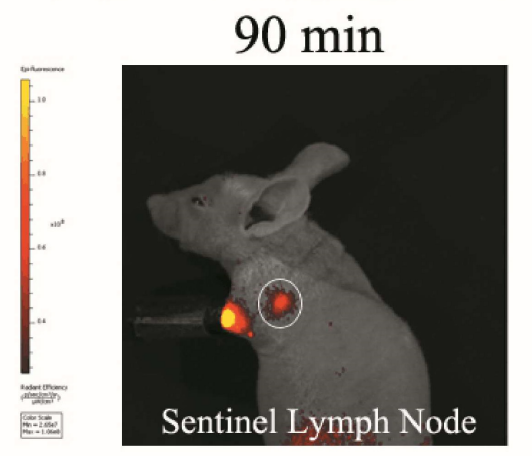

B

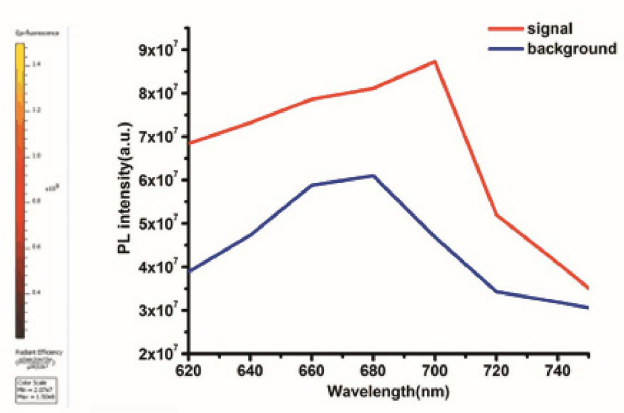

C
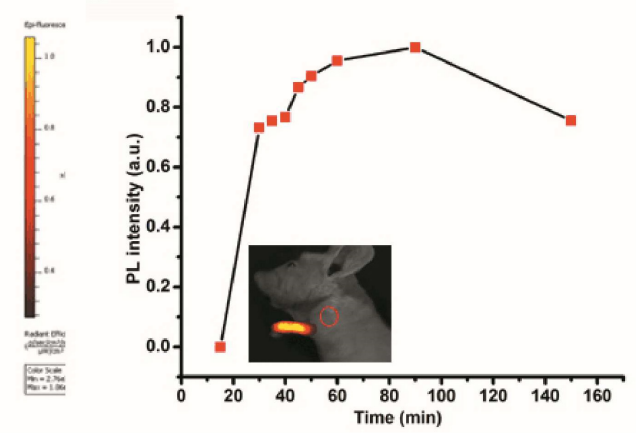

FIGURE 5 | Sentinel lymph node (SLN) imaging of a nude mouse with PS-PEG@TB NPs intradermally injected into the forepaw pad at various times post-injection. (A) Fluorescence imaging (excitation light: $570 \mathrm{~nm}$ ) of the mouse 15, 40, 60 and 90 min after injection. (B) The fluorescence intensity at different wavelengths with signal and background. (C) The fluorescence intensity of SLNs at different times post-injection.

(Supplementary Figure S12G), the blood vessels observed were mainly capillaries. Supplementary Figure $\mathbf{S 1 2 H}$ is a stacked three-photon fluorescence image from a depth of 0 to 635 $\mu \mathrm{m}$. A 3D mixed image of the PS-PEG@TB NPs in the blood vessels of the brain of a mouse was reconstructed as shown in Supplementary Figure S13. Reconstructing from different angles can restore the structure of mouse brain blood vessels to the greatest degree, which is beneficial for the exploration of brain science. The deep tissue imaging capability, high spatial resolution, high signal-to-noise ratio, and low thermal damage to biological samples make 3PL imaging very useful for brain vascular imaging of small animals.

\section{PS-PEG@TB NPs for SLN Mapping of Mice}

The sentinel lymph node (SLN) can prevent lymphatic spreading out, so its clinical significance is very important. Figure 5A shows the SLN of nanoparticle imaging in mice. SLN is located in a white circle. The change in fluorescence intensity at the SLN after different injection times can be observed. Figure 5B shows a comparison of the luminescence signal at the SLN and the fluorescence signal of the other parts of the mouse (autologous background fluorescence). The results showed that the light intensity of the SLN signal is significantly higher than the background fluorescence signal, and it can be confirmed that the nanoparticles actually reached the SLN. Figure $5 \mathrm{C}$ shows the trend of the fluorescence intensity of the SLN after injection at different times. After $30 \mathrm{~min}$, the signal slowly appeared in the SLN. As time went on, the nanoparticles continued to enter the SLN as the body fluid circulated, and the fluorescence intensity reached a maximum after $90 \mathrm{~min}$. The fluorescence signal then began to decay. The signal becomes weak after $150 \mathrm{~min}$. Through signal intensity analysis, this material can be used as a good lymph node contrast agent, which has potential clinical application value.

\section{CONCLUSION}

The properties of these nanoparticles were strongly dependent on the composition of the encapsulated amphiphilic copolymer, where the polystyrene moiety can boost emission effectively compared with other non-aromatic polymeric segments. Hence, by using PS-PEG encapsulation to increase the ratio of the polystyrene moiety, the PS-PEG@TB NPs showed near-infrared emission at $665 \mathrm{~nm}$ with the highest fluorescent quantum yield of $27 \%$ and displayed excellent chemical stability, low toxicity and biocompatibility.

Furthermore, PS-PEG@TB NPs, which possess strong threephoton fluorescence, were utilized as fluorescent contrast agents for 3PL imaging under $1550 \mathrm{~nm}$ laser excitation, and for 
in vivo angiography of a mouse brain and ear, showing good imaging depths of 635 and $180 \mu \mathrm{m}$, respectively. We further demonstrated that these nanoparticles can effectively target the sentinel lymph node (SLN) of mice. This study virtually outlines an efficient encapsulation strategy for fabricating highly emissive organic nanoparticles and a high imaging depth multiphoton fluorescence imaging technique is applied in vivo. We believe that PS-PEG@TB NPs will become a good candidate for clinical deep-tissue bioimaging in the future.

\section{DATA AVAILABILITY STATEMENT}

All datasets presented in this study are included in the article/Supplementary Material.

\section{ETHICS STATEMENT}

The animal experiments were performed strictly in compliance with the requirements and guidelines of the Institutional Ethical Committee of Animal Experimentation of Zhejiang University.

\section{REFERENCES}

Alifu, N., Yan, L., Zhang, H., Zebibula, A., Zhu, Z., Xi, W., et al. (2017). Organic dye doped nanoparticles with NIR emission and biocompatibility for ultra-deep in vivo two-photon microscopy under $1040 \mathrm{~nm}$ femtosecond excitation. Dyes Pigments 143, 76-85. doi: 10.1016/j.dyepig.2017. 04.017

Axelrod, D. (2001). Total internal reflection fluorescence microscopy in cell biology. Traffic 2, 764-774. doi: 10.1034/j.1600-0854.2001.21104.x

Baddeley, D., Crossman, D., Rossberger, S., Cheyne, J. E., Montgomery, J. M., Jayasinghe, I. D., et al. (2011). 4D super-resolution microscopy with conventional fluorophores and single wavelength excitation in optically thick cells and tissues. PLoS One 6:e20645. doi: 10.1371/journal.pone.0020645

Fateminia, S. M., Wang, Z., Goh, C. C., Manghnani, P. N., Wu, W., Mao, D., et al. (2017). Nanocrystallization: a unique approach to yield bright organic nanocrystals for biological applications. Adv. Mater. 29:1604100. doi: 10.1002/ adma.201604100

Feng, G., Liu, J., Liu, R., Mao, D., Tomczak, N., Liu, B., et al. (2017). Ultrasmall conjugated polymer nanoparticles with high specificity for targeted cancer cell imaging. Adv. Sci. 4:1600407. doi: 10.1002/advs.201600407

Kobat, D., Horton, N. G., and Xu, C. (2011). In vivo two-photon microscopy to $1.6-\mathrm{mm}$ depth in mouse cortex. J. Biomed. Opt. 16:106014. doi: 10.1117/1. 3646209

Li, Y., Shao, A., Wang, Y., Mei, J., Niu, D., Gu, J., et al. (2016). Morphology-tailoring of a red aiegen from microsized rods to nanospheres for tumor-targeted bioimaging. Adv. Mater. 28, 3187-3193. doi: 10.1002/adma.201504782

Liu, L.-J., Liu, W., Ji, G., Wu, Z.-Y., Xu, B., Qian, J., et al. (2019). NIR emission nanoparticles based on FRET composed of AIE luminogens and NIR dyes for two-photon fluorescence imaging. Chinese J. Polym. Sci. 37, 401-408. doi: 10.1007/s10118-019-2206-3

Liu, Y., Deng, C., Tang, L., Qin, A., Hu, R., Sun, J. Z., et al. (2011). Specific detection of D-glucose by a tetraphenylethene-based fluorescent sensor. J. Am. Chem. Soc. 133, 660-663. doi: 10.1021/ja107086y

Lu, H., Zheng, Y., Zhao, X., Wang, L., Ma, S., Han, X., et al. (2016). Highly efficient far red/near-infrared solid fluorophores: aggregation-induced emission, intramolecular charge transfer, twisted molecular conformation, and bioimaging applications. Angew. Chem. Int. Ed. Engl. 55, 155-159. doi: 10.1002/ anie. 201507031

\section{AUTHOR CONTRIBUTIONS}

YD and BX: conception and design. JQ and GJ: administrative support. XW and QL: provision of study materials or patients. NA and ZW: collection and assembly of data. JQ and RC: data analysis and interpretation. DS: manuscript writing. All authors approved the final manuscript.

\section{FUNDING}

This work was supported by the Natural Science Foundation of China (81773171) and the Science and Technology Department of Jilin Province (20170311005YY, 20200404197YY, and 20200201349JC).

\section{SUPPLEMENTARY MATERIAL}

The Supplementary Material for this article can be found online at: https://www.frontiersin.org/articles/10.3389/fbioe. 2020.01029/full\#supplementary-material

Ma, K., Wang, H., Li, H., Xu, B., and Tian, W. (2017). Label-free detection for SNP using AIE probes and carbon nanotubes. Sens. Actuators B Chem. 253, 92-96. doi: 10.1016/j.snb.2017.06.055

Massin, J., Dayoub, W., Mulatier, J.-C., Aronica, C., Bretonnière, Y., and Andraud, C. (2011). Near-infrared solid-state emitters based on isophorone: synthesis, crystal structure and spectroscopic properties. †. Chem. Mater. 23, 862-873. doi: $10.1021 / \mathrm{cm} 102165 \mathrm{r}$

Ni, J. S., Zhang, P., Jiang, T., Chenm, Y., Su, H., Wang, D., et al. (2018). Red/NIR-Emissive Benzo[d]imidazole-cored AIEgens: facile molecular design for wavelength extending and in vivo tumor metabolic imaging. Adv. Mater. 30:e1805220. doi: 10.1002/adma.201805220

Qi, J., Chen, C., Zhang, X., Hu, X., Ji, S., Kwok, R. T. K., et al. (2018). Light-driven transformable optical agent with adaptive functions for boosting cancer surgery outcomes. Nat. Commun. 9:1848. doi: 10.1038/s41467-018-04222-8

Qi, Q., Li, Y., Yan, X., Zhang, F., Jiang, S., Su, J., et al. (2016). Intracellular pH sensing using polymeric micelle containing tetraphenylethylene-oxazolidine. Polym. Chem. 7, 5273-5280. doi: 10.1039/c6py01072e

Qian, G., Zhong, Z., Luo, M., Yu, D., Zhang, Z., Wang, Z. Y., et al. (2009). Simple and efficient near-infrared organic chromophores for light-emitting diodes with single electroluminescent emission above $1000 \mathrm{~nm}$. Adv. Mater. 21, 111-116. doi: 10.1002/adma.200801918

Qian, J., and Tang, B. Z. (2017). AIE luminogens for bioimaging and theranostics: from organelles to animals. Chem 3, 56-91. doi: 10.1016/j.chempr.2017.05.010

Qian, J., Wang, D., Cai, F. H., Xi, W., Peng, L., Zhu, Z. F., et al. (2012). Observation of multiphoton-induced fluorescence from graphene oxide nanoparticles and applications in in vivo functional bioimaging. Angew. Chem. Int. Ed. Engl. 51, 10570-10575. doi: 10.1002/anie.201206107

Rust, M. J., Bates, M., and Zhuang, X. (2006). Sub-diffraction-limit imaging by stochastic optical reconstruction microscopy (STORM). Nat. Methods 3, 793-795. doi: 10.1038/nmeth929

Shao, A., Xie, Y., Zhu, S., Guo, Z., Zhu, S., Guo, J., et al. (2015). Far-Red and NearIR AIE-active fluorescent organic nanoprobes with enhanced tumor-targeting efficacy: shape-specific effects. Angew. Chem. Int. Ed. Engl. 54, 7275-7280. doi: 10.1002/anie.201501478

Shi, H., Kwok, R. T., Liu, J., Xing, B., Tang, B. Z., and Liu, B. (2012). Real-time monitoring of cell apoptosis and drug screening using fluorescent light-up probe with aggregation-induced emission characteristics. J. Am. Chem. Soc. 134, 17972-17981. doi: 10.1021/ja3064588 
Situ, B., Chen, S., Zhao, E., Leung, C. W. T., Chen, Y., Hong, Y., et al. (2016). Real-time imaging of cell behaviors in living organisms by a mitochondriatargeting AIE fluorogen. Adv. Funct. Mater. 26, 7132-7138. doi: 10.1002/adfm. 201602865

Song, Z., Mao, D., Sung, S. H., Kwok, R. T., Lam, J. W., Kong, D., et al. (2016). Activatable fluorescent nanoprobe with aggregation-induced emission characteristics for selective in vivo imaging of elevated peroxynitrite generation. Adv. Mater. 28, 7249-7256. doi: 10.1002/adma.201601214

Wang, D., Qian, J., He, S., Park, J. S., Lee, K. S., Han, S., et al. (2011). Aggregationenhanced fluorescence in PEGylated phospholipid nanomicelles for in vivo imaging. Biomaterials 32, 5880-5888. doi: 10.1016/j.biomaterials.2011.04.080

Wang, H., Ma, K., Xu, B., and Tian, W. (2016). Tunable supramolecular interactions of aggregation-induced emission probe and graphene oxide with biomolecules: an approach toward ultrasensitive label-free and "Turn-On" DNA sensing. Small 12, 6613-6622. doi: 10.1002/smll.201601544

Wang, Y., Chen, M., Alifu, N., Li, S., Qin, W., Qin, A., et al. (2017). Aggregationinduced emission luminogen with deep-red emission for through-skull threephoton fluorescence imaging of mouse. ACS Nano 11, 10452-10461. doi: 10. 1021/acsnano.7b05645

Wang, Z., Xu, B., Zhang, L., Zhang, J., Ma, T., Zhang, J., et al. (2013). Folic acidfunctionalized mesoporous silica nanospheres hybridized with AIE luminogens for targeted cancer cell imaging. Nanoscale 5, 2065-2072. doi: 10.1039/ c2nr33685e

Wu, W., Mao, D., Hu, F., Xu, S., Chen, C., Zhang, C. J., et al. (2017). A highly efficient and photostable photosensitizer with near-infrared aggregationinduced emission for image-guided photodynamic anticancer therapy. $A d v$. Mater. 29:1700548. doi: 10.1002/adma.201700548
Yan, L., Zhang, Y., Xu, B., and Tian, W. (2016). Fluorescent nanoparticles based on AIE fluorogens for bioimaging. Nanoscale 8, 2471-2487. doi: 10.1039/ c5nr05051k

Zhang, Y., Chang, K., Xu, B., Chen, J., Yan, L., Ma, S., et al. (2015). Highly efficient near-infrared organic dots based on novel AEE fluorogen for specific cancer cell imaging. RSC Adv. 5, 36837-36844. doi: 10.1039/c5ra04669f

Zhen, S., Wang, S., Li, S., Luo, W., Gao, M., Ng, L. G., et al. (2018). Efficient Red/Near-infrared fluorophores based on Benzo[1,2-b:4,5-b']dithiophene 1,1,5,5-tetraoxide for targeted photodynamic therapy and in vivo two-photon fluorescence bioimaging. Adv. Funct. Mater. 28:1706945. doi: 10.1002/adfm. 201706945

Zheng, Z., Zhang, T., Liu, H., Chen, Y., Kwok, R. T. K., Ma, C., et al. (2018). Bright near-infrared aggregation-induced emission luminogens with strong twophoton absorption, excellent organelle specificity, and efficient photodynamic therapy potential. ACS Nano 12, 8145-8159. doi: 10.1021/acsnano.8b03138

Conflict of Interest: The authors declare that the research was conducted in the absence of any commercial or financial relationships that could be construed as a potential conflict of interest.

Copyright (c) $2020 \mathrm{Du}$, Alifu, Wu, Chen, Wang, Ji, Li, Qian, Xu and Song. This is an open-access article distributed under the terms of the Creative Commons Attribution License (CC BY). The use, distribution or reproduction in other forums is permitted, provided the original author(s) and the copyright owner(s) are credited and that the original publication in this journal is cited, in accordance with accepted academic practice. No use, distribution or reproduction is permitted which does not comply with these terms. 